A Antoine Kazzi
Emergency Medicine
University of California,
Irvine
Orange, CA 92868
Correspondence to:
Dr Kazzi
akazzi@uci.edu
Competing interests:
None declared
West J Med
$2000 ; 172: 388-389$

\title{
COMMENTARY Give emergency medicine true departmental control
}

As early as 1958 , confronted by a $400 \%$ rise in emergency department (ED) visits in 15 years, the medical community called for a modernization of the staffing patterns in emergency rooms. ${ }^{1}$ This critical mass of need resulted in the establishment of the first "ER" and emergency physician group in 1961. Over the next 2 decades, emergency medical services developed, and emergency medicine became a specialty with its own training and board certification.

In 1966, the American Medical Assocation reviewed the increased use, staffing patterns, and quality of care in EDs and recommended "the recognition of the emergency service as a department" as "the first step in solving its problems." 2

Since these early days, the number of patients attending EDs has continued to grow as much as $2 \%$ annually. In 1995, 100 million patients visited EDs in the United States. This huge increase occurred despite aggressive legislative and economic strategies led by managed care that aimed to shift patients away from EDs to their primary care physicians.

In 2000, Richards and colleagues report that ED overcrowding is once again a serious problem. The authors attribute it to many factors, most of which they think are beyond the control of EDs.

The recognition of the emergency service as a hospital department should be genuine and comprehensive. This must involve giving it true control over 3 key areas: decision making, financing, and maintaining efficiency.
The ED leadership should be able to directly monitor and alter the processes of patient registration and hospital admission. Access to emergency physicians, treatment delivery, and transfer to a floor or unit bed should not be delayed by, for example, the need for preauthorization. Managers of EDs should be empowered to monitor and seek solutions to lengthy delays in the provision of studies and their interpretations. Legislation is needed to mandate that adequate screening and stabilization are provided to patients before any preauthorization of care. Managed care gatekeepers are unable to reliably address the urgency of a complaint by telephone and should not second-guess the judgment of a treating emergency physician. Current law restricts the primary responsibility for the quality and outcome to the emergency physician and treating facility. Gatekeepers remain legally unaccountable for pressures they exert during preauthorization calls, despite inherent financial conflicts of interest. Legislation should prohibit prolonged authorization processes for poststabilization care, such as admission or specialized consultations. Emergency physicians should be enabled to designate the service to which a patient is admitted and to mobilize without delay emergent consultations they deem necessary. Managed care plans and hospitals must provide clear-cut on-call panels for all patients, regardless of their funding status.

Professional and facility charges for care provided in an ED should never be "bundled" with those of the inpatient care phase. Capitation rates for ED services should not be 
bundled with primary care groups who are using them. Both forms of bundling will result in "reverse utilization," a process of convenient overuse of the ED safety net, which is mandated by federal law to see and provide care for all unscheduled patients. Emergency department resources and revenue must not be shifted away through taxation or management schemes that exceed fair market value. They are not meant for non-ED-related expenses or for profit-driven management corporations. They are needed within EDs to reduce overcrowding and to provide optimal care. Laws restricting the corporate practice of medicine should be enforced. Physicians and nurses provide patient care; they are not businesspeople.

To reduce costs, hospitals and insurers shift timeconsuming tasks to the ED for each patient visit. New tasks have also been added, such as superfluous documentation and computerized order entries. Such "right-sizing" increases the time needed per patient, compounding the overcrowding. Staffing and resources should not be allocated based on average patient flow. A safety margin must be provided for the often-unpredictable peaks of patient volume and urgency. Finally, ED physician and nursing administrators should maintain clinical involvement to experience directly the results of their decisions.

Future population growth and aging will only worsen ED overcrowding. The right-sizing of emergency medicine and the shifting or bundling of its revenue are not new. They are, however, growing trends in this dynamic "marketplace." Doing more with less will no doubt compromise the quality of care for all our patients.

References

1 Shorrliffe EC. The ER and the changing patterns of medical care. $N$ Engl J Med 1958;258:20-25.

2 AMA Department of Hospitals and Medical Facilities. The emergency department problem: an overview. JAMA 1966;198:380-383.

\section{Practice point}

\section{Keep looking for a reason}

Sarah was 22 when she presented with the dreadful signs of a large space-occupying lesion deep in the dominant parietal lobe. She was drowsy with a severe headache, had severe dysphasia, and there was a signficant right-sided weakness. The CT scan said it all; a malignant intrinsic tumor at the trigone of the lateral ventricle, probably a glioblastoma.

She rallied a little on steroids overnight and so I performed a craniotomy, having suitably warned her worried family of the likely outcome and prognosis. I found a very bloody tumor that had some definition from the surrounding brain. The pathologist told me that the frozen section showed a highly malignant brain tumor, consistent with the radiologist's diagnosis.

The survival from this type of tumor in this position is measured in months, and there is no evidence that surgery does much more than relieve the pressure symptoms. Consequently, I completed a cautious internal decompression, stopped the bleeding, and closed up. She was unchanged, but bled into the tumor remnant the following day and I had to reopen the craniotomy. That night, I was relieved that she was no worse. Sarah's tumor was a gliosarcoma. This is a rare and even more malignant variant of a glioblastoma, in which it is thought that the new vessel-forming factors released by the primary brain tumor induce sarcomatous change in the blood vessels. The news was not good.

Surprisingly, Sarah started to get better. Her speech improved and her hemiparesis almost disappeared. She was referred for radiotherapy and underwent the full 6 weeks' course. The follow-up scan also looked good. She lost her job in an insurance company, but she became a police receptionist.

Two years went by, but there was no sign of the tumor on further scans. By then, Sarah had become a good friend of the hospital, active in collecting money for the development fund, and talking to the press about her treatment.

After 3 years, when the vast majority of patients with a glioblastoma are dead, I asked the pathologists to review the case, but despite the tumor's unusual behaviour, they could not come up with any other diagnosis.

Meanwhile, Sarah had married and was thinking of starting a family. By this time, much more sensitive magnetic resonance imaging had become more freely available, and the scan showed no recurrence. Six years after her initial diagnosis, I asked the pathologist to rereview the case. By now, immunocytochemistry was much more sophisticated, and despite the mitoses and other seemingly malignant features, he was able to reclassify the lesion as a much more benign pleomorphic xanthoastrocytoma.

When I told Sarah this exciting news, I was most surprised that it seemed unimportant to her, almost to the point of disinterestedness.

She remains well almost 10 years from when her initial diagnosis was rendered. Her scan is clear, but she still worries about recurrence. I worry about the possibility of radionecrosis, which sometimes afflicts long term survivors of brain irradiation.

I have learned 3 things from Sarah's case. First, that having lived with the diagnosis of having had cancer but being free from recurrence, being an unusual statistic with a semibenign condition of unknown behavior, lacks meaning. Second, when tumors behave in highly unusual ways, keep looking for a reason. Third, do not expect other patients' tumors to behave in a similarly unusual fashion-a temptation that I fell into at least once.

Michael Powell, London 